\title{
Intrinsic metrological resolution as a distance measure and nonclassical light
}

\author{
Ángel Riva: $:$ \\ School of Physics, Astronomy and Mathematics, University of Hertfordshire, \\ College Lane, Hatfield, Herts, AL10 9AB, United Kingdom

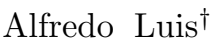 \\ Departamento de Óptica, Facultad de Ciencias Físicas, \\ Universidad Complutense, 28040 Madrid, Spain
}

(Dated: June 12, 2008)

\begin{abstract}
We elaborate on a Hilbert-Schmidt distance measure assessing the intrinsic metrological accuracy in the detection of signals imprinted on quantum probe states by signal-dependent transformations. For small signals this leads to a probe-transformation measure $\Lambda$ fully symmetric on the probe $\rho$ and the generator $G$ of the transformation $\Lambda(\rho, G)=\Lambda(G, \rho)$. Although $\Lambda$ can be regarded as a generalization of variance we show that no uncertainty relation holds for the product of measures corresponding to complementary generators. We show that all states with resolution larger than coherent states are nonclassical. We apply this formalism to feasible probes and transformations.

PACS numbers: 42.50.St, 42.50.Dv, 03.65.-w
\end{abstract}

\section{INTRODUCTION}

Quantum metrology is a key issue of quantum mechanics involving fundamental concepts such as uncertainty relations, complementarity, and nonclassical properties. The basic objective of quantum metrology is to infer the value of a given unknown signal $\chi$ as accurately as possible at minimum cost. Signals $\chi$ are encoded on quantum states by $\chi$-dependent unitary transformations $U_{\chi}$ acting on an input probe state $\rho$, so that all the information about $\chi$ is contained in the output probe state $\rho_{\chi}=U_{\chi} \rho U_{\chi}^{\dagger}$

The intrinsic accuracy of the detection depends primarily on the contribution of two independent factors: the preparation of the probe $\rho$ and the encoding transformation $U_{\chi}$. While most works on quantum metrology focus on the optimization of the input probe state $\rho$ [1, 2], recently it has been put forward the advantages of investigating optimal encoding transformations $U_{\chi}$ allowing more robust and accurate detection schemes [3] .

In this work we elaborate on the assessment of the intrinsic accuracy by using the Hilbert-Schmidt distance between $\rho$ and $\rho_{\chi}[4,[5,6,6,6]$. More specifically, we show that:

(i) For small signals the Hilbert-Schmidt distance becomes a probe-transformation measure $\Lambda$ fully symmetric on the probe $\rho$ and the generator $G$ of the transformation $\Lambda(\rho, G)=\Lambda(G, \rho)$. This acquires the form of a generalization of variance previously used in quantum mechanics and classical optics [9, 10] (Sec. II).

(ii) We derive new expressions for the probe-generator measure $\Lambda(\rho, G)$ (Sec. II).

\footnotetext{
*Electronic address: A.Rivas@herts.ac.uk

$\dagger$ Electronic address:

URL: http://www.ucm.es/info/gioq
}

alluis@fis.ucm.es (iii) We demonstrate that $\Lambda(\rho, G)$ is always bounded from above by variance (Sec. II).

(iv) Despite resembling a generalization of variance, we show that no uncertainty relation holds for the product $\Lambda(\rho, A) \Lambda(\rho, B)$ for complementary generators $A, B$ (Sec. II).

(v) We determine optimum generators leading to maximum resolution for fixed input probe states.

(vi) We show that the probe-generator measure $\Lambda(\rho, G)$ predicts nonclassical behavior (in the sense of lack of positive phase-space representative $P(\alpha)$ [11] ) for all states providing larger resolution than coherent states (Sec. III).

(vii) We apply this formalism to feasible Gaussian probes and standard transformations, determining the probes reaching optimum intrinsic resolution (Sec. IV).

\section{INTRINSIC METROLOGICAL RESOLUTION}

The signal to be detected $\chi$ is encoded in the input probe state $\rho$ by a unitary transformation. For definiteness, we focus on the most common and practical case of constant generators $G$ independent of the parameter $\chi$, $U_{\chi}=\exp (i \chi G)$,

$$
\rho_{\chi}=\exp (i \chi G) \rho \exp (-i \chi G),
$$

where $G$ is the Hermitian generator of the transformation. The intrinsic accuracy is given first and foremost by the distinguishability between $\rho$ and $\rho_{\chi}$. A convenient measure of distinguishability is the Hilbert-Schmidt distance

$$
d_{H S}^{2}(\chi)=\operatorname{tr}\left[\left(\rho-\rho_{\chi}\right)^{2}\right]=\operatorname{tr}\left(\rho^{2}\right)+\operatorname{tr}\left(\rho_{\chi}^{2}\right)-2 \operatorname{tr}\left(\rho \rho_{\chi}\right) .
$$

The overlap term, $\operatorname{tr}\left(\rho \rho_{\chi}\right)$, represents the survival probability, expressing the inertia of $\rho$ to the changes generated 
by $G$. There are many distance measures that may be used leading to largely equivalent results. Among them, the Hilbert-Schmidt distance is selected here because of conceptual and computational simplicity, and its proximity with experimental procedures [7].

All similar measures of distinguishability between density matrices $\rho$ and $\rho_{\chi}$ that may be used, such as relative entropy, trace distance, Bures distance, Hellinger distance, have drawbacks, such as not leading to proper distance measures (relative entropy), lack of physical interpretation or rather complex evaluation procedures (Hellinger and Bures distances). Further comparison of properties can be found in Ref. [4]. In this regard, the main drawback of the Hilbert-Schmidt distance quoted in the literature is the no-monotony decrease under quantum operations. As discussed in Ref. [8] this is a trouble to use this distance to quantify entanglement when constructing entanglement monotones. More properly, only monotonicity under local operations and classical communication (LOCC) is needed, and general monotonicity is a sufficient condition. Monotonicity is satisfied by distinguishability measures such as relative entropy and Bures distance [8].

Let us explain why monotonicity is not an essential property in our context so that the Hilbert-Schmidt distance can be safely used for our purposes. Monotonicity of a distance measure $d\left(\rho_{1}, \rho_{2}\right)$ means that $d\left(\rho_{1}, \rho_{2}\right) \geq d\left[\mathcal{E}\left(\rho_{1}\right), \mathcal{E}\left(\rho_{2}\right)\right]$ where $\mathcal{E}$ are quantum operations (completely positive trace-preserving maps) that include LOCC as a particular case. The key point is that this property is always discussed in terms of completely independent density matrices $\rho_{1,2}$. However, in our case $\rho_{1,2}$ are not independent, since one of them is the result of a signal-dependent transformation $\mathcal{K}$ acting on the other $\rho_{2}=\mathcal{K}\left(\rho_{1}\right)$. Since in general $\mathcal{E}[\mathcal{K}(\rho)] \neq \mathcal{K}[\mathcal{E}(\rho)]$ there is no point comparing $d[\rho, \mathcal{K}(\rho)]$ and $d\{\mathcal{E}(\rho), \mathcal{E}[\mathcal{K}(\rho)]\}$, since the last one is not of the form $d\left[\rho^{\prime}, \mathcal{K}\left(\rho^{\prime}\right)\right]$. In physical terms $\mathcal{E}$ cannot act on $\rho$ and $\mathcal{K}(\rho)$ simultaneously, since $\rho$ and $\mathcal{K}(\rho)$ never coexist (the former precedes the latter). In other words, in our case the distance $d$ must be understood as a function of $U_{\chi}$ and $\rho$ so that the application of other transformations is out of the scope of our problem.

\section{A. Probe-generator measure}

Concerning metrological applications we are mostly interested in very weak signals so we may consider the limit $\chi \rightarrow 0$. Considering a power series for $d_{H S}^{2}(\chi)$, the first nonvanishing term is

$$
d_{H S}^{2}(\chi) \simeq 2 \chi^{2} \Lambda^{2}(\rho, G)
$$

where the probe-generator functional $\Lambda^{2}(\rho, G)$ is

$$
\Lambda^{2}(\rho, G)=\operatorname{tr}\left(\rho^{2} G^{2}\right)-\operatorname{tr}(\rho G \rho G) .
$$

Therefore $\Lambda(\rho, G)$ measures the capability of $G$ to efficiently imprint small signals on the input probe $\rho$, so that larger $\Lambda^{2}(\rho, G)$ implies larger resolution. The performance measure (2.3) can be regarded as a generalization of the more familiar estimation uncertainty $\delta \chi[1,2]$

$$
\delta \chi \geq \frac{1}{2 \Delta_{\rho} G}
$$

where $\Delta_{\rho} G$ is the variance

$$
\left(\Delta_{\rho} G\right)^{2}=\operatorname{tr}\left(\rho G^{2}\right)-[\operatorname{tr}(\rho G)]^{2} .
$$

The probe-generator measure $\Lambda(\rho, G)$ can be regarded as a generalization of variance since for pure states $\rho^{2}=$ $\rho=|\psi\rangle\langle\psi|$ we have [10]

$$
\Lambda^{2}(|\psi\rangle, G)=\left\langle\psi\left|G^{2}\right| \psi\right\rangle-\langle\psi|G| \psi\rangle^{2}=\left(\Delta_{\psi} G\right)^{2} .
$$

However a literal strict interpretation of $\Lambda^{2}(\rho, G)$ as an uncertainty measure is questionable, or even misleading, as shown below.

Let us note the complete symmetry between $\rho$ and $G$, $\Lambda(\rho, G)=\Lambda(G, \rho)$. This symmetry suits to the idea of the joint accountability of probe and transformation to metrological performance.

\section{B. Equivalent expressions}

The probe-generator functional $\Lambda$ can be expressed also as

$$
\Lambda^{2}(\rho, G)=-\frac{1}{2} \operatorname{tr}\left([\rho, G]^{2}\right)=\frac{1}{2} \operatorname{tr}\left[\left(\frac{d \rho_{\chi}}{d \chi}\right)_{\chi=0}^{2}\right] .
$$

This can be regarded as the analog of the Wigner-Yanase skew information after replacing $\sqrt{\rho}$ by $\rho$ [4, 5].

In terms of the spectrum and statistics of $G$ the following expression holds

$$
\Lambda^{2}(\rho, G)=\frac{1}{2} \sum_{j, k}\left(g_{j}-g_{k}\right)^{2}\left|\left\langle g_{k}|\rho| g_{j}\right\rangle\right|^{2},
$$

where $G\left|g_{j}\right\rangle=g_{j}\left|g_{j}\right\rangle$. This has been used in classical optics to asses effective spatial correlations of light beams [9].

According to the full symmetry between $G$ and $\rho$ we can derive a relation dual to Eq. (2.9) in terms of the spectrum of $\rho$

$$
\Lambda^{2}(\rho, G)=\frac{1}{2} \sum_{j, k}\left(r_{j}-r_{k}\right)^{2}\left|\left\langle r_{k}|G| r_{j}\right\rangle\right|^{2},
$$

where $\left|r_{j}\right\rangle$ is the orthonormal basis defined by the eigenvectors of $\rho, \rho\left|r_{j}\right\rangle=r_{j}\left|r_{j}\right\rangle$, including those with vanishing eigenvalue.

Furthermore, $\Lambda(\rho, G)$ can be also related with a kind of weighted version of variances of weak values. This can be seen after expressing $\rho$ in the $P(\alpha)$ representation

$$
\rho=\int d^{2} \alpha P(\alpha)|\alpha\rangle\langle\alpha|,
$$


where $|\alpha\rangle$ are coherent states [11]. Using this representation in Eq. (2.4) we get

$$
\begin{aligned}
\Lambda^{2}(\rho, G)= & \int d^{2} \alpha d^{2} \beta P(\alpha) P(\beta)|\langle\alpha \mid \beta\rangle|^{2} \\
& \times\left[G^{(2)}(\alpha, \beta)-\left|G^{(1)}(\alpha, \beta)\right|^{2}\right],
\end{aligned}
$$

where $G^{(k)}(\alpha, \beta)$ is the weak value of $G^{k}$ in the coherent states $|\alpha\rangle,|\beta\rangle[12]$

$$
G^{(k)}(\alpha, \beta)=\frac{\left\langle\beta\left|G^{k}\right| \alpha\right\rangle}{\langle\beta \mid \alpha\rangle} .
$$

Further expressions for $\Lambda^{2}(\rho, G)$ can be obtained for Cartesian conjugate variables in terms of the Wigner function [10].

\section{Variance bound}

Using Eq. (2.9) we can demonstrate that $\Lambda$ is always bounded from above by variance

$$
\Lambda^{2}(\rho, G) \leq\left(\Delta_{\rho} G\right)^{2},
$$

the equality being reached for pure states. To this end we note that the density matrices $\rho$ and $\sigma$ have the same variance $\Delta_{\rho} G=\Delta_{\sigma} G$, where $\sigma$ is a pure state $\sigma=|\psi\rangle\langle\psi|$ with

$$
|\psi\rangle=\sum_{k} \sqrt{\left\langle g_{k}|\rho| g_{k}\right\rangle} e^{i \varphi_{k}}\left|g_{k}\right\rangle,
$$

where $\varphi_{k}$ are phases. Thus, by construction $\sigma$ is positive and Hermitian, and it holds

$$
\left|\left\langle g_{k}|\sigma| g_{j}\right\rangle\right|^{2}=\left\langle g_{k}|\rho| g_{k}\right\rangle\left\langle g_{j}|\rho| g_{j}\right\rangle .
$$

The equality $\Delta_{\rho} G=\Delta_{\sigma} G$ holds because the variance of $G$ depends exclusively on the diagonal matrix elements, that are equal for both density matrices $\left\langle g_{k}|\rho| g_{k}\right\rangle=$ $\left\langle g_{k}|\sigma| g_{k}\right\rangle$. Furthermore, the positivity of $\rho$ implies that

$$
\left|\left\langle g_{k}|\rho| g_{j}\right\rangle\right|^{2} \leq\left\langle g_{k}|\rho| g_{k}\right\rangle\left\langle g_{j}|\rho| g_{j}\right\rangle=\left|\left\langle g_{k}|\sigma| g_{j}\right\rangle\right|^{2} .
$$

Thus, from Eqs. (2.7), (2.9), and (2.17) we get

$$
\Lambda^{2}(\rho, G) \leq \Lambda^{2}(\sigma, G)=\left(\Delta_{\sigma} G\right)^{2}=\left(\Delta_{\rho} G\right)^{2},
$$

which demonstrates Eq. (2.14).

\section{Lack of uncertainty relation}

The dependence of $\Lambda(\rho, G)$ on the coherence terms $\left\langle g_{k}|\rho| g_{j}\right\rangle$ reveals that this is more than a measure of fluctuations. In this regard, for example, we have $\Lambda(\rho, G)=$ 0 if and only if

$$
\rho=\sum_{j} p_{j}\left|g_{j}\right\rangle\left\langle g_{j}\right|
$$

with $p_{j} \geq 0$ and $\sum_{j} p_{j}=1$, so that $\Delta_{\rho} G$ can take any value depending on $p_{j}$. This is further discussed in Sec. IVC.

Moreover, despite that $\Lambda$ resembles a generalization of variance, it does not lead to any uncertainty relation when applied to complementary observables. More specifically we show that there is no lower bound for the product $\Lambda(\rho, X) \Lambda(\rho, Y)$, where $X, Y$ are two Cartesian conjugate observables, analogous to position and linear momentum,

$$
X=\frac{1}{\sqrt{2}}\left(a+a^{\dagger}\right), \quad Y=\frac{i}{\sqrt{2}}\left(a^{\dagger}-a\right),
$$

with $[X, Y]=i$, and $a^{\dagger}, a$ are creation and annihilation operators with $\left[a, a^{\dagger}\right]=1$. In such a case, we show in more detail in Sec. IVA that for the squeezed vacuum states (4.5) we get

$$
\Lambda^{2}(\rho, X)=\frac{1}{8 \Delta X(\Delta Y)^{3}}, \quad \Lambda^{2}(\rho, Y)=\frac{1}{8 \Delta Y(\Delta X)^{3}},
$$

so that

$$
\Lambda(\rho, X) \Lambda(\rho, Y)=\frac{1}{8(\Delta X)^{2}(\Delta Y)^{2}} .
$$

There is no lower bound for this product since $\Lambda(\rho, X) \Lambda(\rho, Y) \rightarrow 0$ when $\Delta X \rightarrow \infty$ or $\Delta Y \rightarrow \infty$. Moreover, we can notice that we can have $\Lambda(\rho, X) \rightarrow 0$ and $\Lambda(\rho, Y) \rightarrow 0$ simultaneously.

One may wonder whether the lack of uncertainty relation is related with the lack of monotonicity of the Hilbert-Schmidt distance. At first qualitative argument, we note that, roughly speaking, lack of monotonicity is related with increase of distance, while lack of uncertainty relation is given by the fully opposite effect, i. e., decreasing distances. Anyway a simple and expeditious procedure to solve this question is to show that the lack of uncertainty relation also occurs for a probetransformation measure (quantum Fisher information) derived from a monotonic distance measure [4, 13, 14]

$$
I_{F}(\rho, G)=\frac{1}{2} \sum_{j, k} \frac{\left(r_{j}-r_{k}\right)^{2}}{r_{j}+r_{k}}\left|\left\langle r_{j}|G| r_{k}\right\rangle\right|^{2},
$$

where as in Eq. (2.10) $\left|r_{j}\right\rangle$ are the eigenvectors of $\rho$ with eigenvalues $r_{j}$, and the sum includes the cases with $r_{j}+r_{k} \neq 0$. This measure is the infinitesimal local form of the Bures distance [4, 13]

$$
d_{B}^{2}\left(\rho_{1}, \rho_{2}\right)=2\left\{1-\operatorname{tr}\left[\left(\rho_{1}^{1 / 2} \rho_{2} \rho_{1}^{1 / 2}\right)^{1 / 2}\right]\right\},
$$

that fulfills monotonicity [8]. Let us compute $I_{F}(\rho, X)$ and $I_{F}(\rho, Y)$ for the quadratures (2.20) in the thermal state

$$
\rho=(1-\xi) \sum_{n=0}^{\infty} \xi^{n}|n\rangle\langle n|
$$


where $\xi<1$ is a real parameter, and $|n\rangle$ are the number states $a^{\dagger} a|n\rangle=n|n\rangle$. After a simple calculation it can be seen that

$$
I_{F}(\rho, X)=I_{F}(\rho, Y)=\frac{1-\xi}{2(1+\xi)} .
$$

Therefore, when $\xi \rightarrow 1$ we get $I_{F}(\rho, X) I_{F}(\rho, Y) \rightarrow 0$ demonstrating that the lack of uncertainty relation is not a consequence of the lack of monotonicity.

On the other hand, there are uncertainty relations involving the product of $\Lambda(\rho, A)$ for one observable with a different measure $\tilde{\Lambda}(\rho, B) \neq \Lambda(\rho, B)$ for the other one [10], such as

$$
\tilde{\Lambda}^{2}(\rho, G)=\frac{1}{2} \sum_{j, k}\left(g_{j}+g_{k}\right)^{2}\left|\left\langle g_{k}|\rho| g_{j}\right\rangle\right|^{2} .
$$

Related uncertainty relations has been proposed in Refs. [4, 15].

Seemingly Ref. [9] introduces a lower bound for the product of the same measure $\Lambda$ for Cartesian conjugate variables within a classical optics framework. However, a closer inspection reveals that such a bound is actually another example of the unbalanced case in Ref. [10] with different measures. Moreover, a balanced uncertainty relation might be seemingly derived from the following formula in Ref. [5]

$$
4 I_{W}(\rho, A) I_{W}(\rho, B) \geq|\operatorname{tr}(\rho[A, B])|^{2},
$$

where $I_{W}(\rho, A)=\Lambda^{2}(\sqrt{\rho}, A)$ is the Wigner-Yanase skew information which is the local infinitesimal form of the Hellinger distance [4, 14]

$$
d_{H}\left(\rho_{1}, \rho_{2}\right)=\operatorname{tr}\left[\left(\rho_{1}^{1 / 2}-\rho_{2}^{1 / 2}\right)^{2}\right] .
$$

However, Eq. (2.28) does not hold [16], as revealed by a simple counterexample since Eq. (2.28) is violated by

$$
\rho=\left(\begin{array}{cc}
0.75 & 0 \\
0 & 0.25
\end{array}\right),
$$

for $A=\sigma_{x}$ and $B=\sigma_{y}$, where $\sigma_{x, y}$ are the corresponding Pauli matrices. In this case we have $I_{W}\left(\rho, \sigma_{x}\right)=$ $I_{W}\left(\rho, \sigma_{y}\right)=0.134$ while $\left|\operatorname{tr}\left(\rho\left[\sigma_{x}, \sigma_{y}\right]\right)\right|^{2}=1$. The lack of a true uncertainty relation for this measure can be further demonstrated again by computing $I_{W}(\rho, X)$ and $I_{W}(\rho, Y)$ for the quadratures (2.20) in the thermal state (2.25), leading to

$$
I_{W}(\rho, X)=I_{W}(\rho, Y)=\frac{1-\sqrt{\xi}}{2(1+\sqrt{\xi})},
$$

so that $I_{W}(\rho, X) I_{W}(\rho, Y) \rightarrow 0$ when $\xi \rightarrow 1$. This agrees with the general relation $I_{W}(\rho, A) \leq I_{F}(\rho, A)$ [14].

Finally, it is worth noting that there are fluctuation measures that seem to defy the existence of an uncertainty relation for complementary observables, as shown in Ref. [17]. Nevertheless, note that $\Lambda \rightarrow 0$ does not mean in this context arbitrary precision. On the contrary, it means complete lack of measuring resolution in the form of indistinguishability between input $\rho$ and transformed $\rho_{\chi}$ states. We recall that as shown in this work $\Lambda$ is a measure of intrinsic resolution rather than a measure of uncertainty.

\section{E. Optimum generators}

The full symmetry between states and transformations invites to look for the optimum generator $G$ leading to maximum intrinsic resolution for fixed probe state $\rho$. This is the dual of the most common operation in quantum metrology of determining the optimum $\rho$ for fixed $G$ 1.].

Despite the symmetry between $\rho$ and $G$ these two operators belong to different classes, unit-trace Hermitian positive definite for $\rho$, and just Hermitian for $G$. Therefore, in order to fully exploit the $\rho, G$ symmetry in the above calculus we restrict ourselves to finite-trace Hermitian positive definite generators. This implies no loss of generality for finite-dimensional $G$ or $\rho$, since finite dimension guarantees finite trace and also positivity by adding a constant to $G$ without altering neither $U$ nor $\Lambda$.

In such a case $\rho$ and $G$ can safely exchange their roles in the above calculus so that the variance bound (2.14) leads us to consider pure generators of the form $G \propto|\psi\rangle\langle\psi|$ where $|\psi\rangle$ is a normalized vector to be determined by the condition of maximum variance of the $\rho$ operator

$$
\left(\Delta_{\psi} \rho\right)^{2}=\left\langle\psi\left|\rho^{2}\right| \psi\right\rangle-\langle\psi|\rho| \psi\rangle^{2} .
$$

Maximum variance is given by the extremal dichotomic statistics provided by states of the form

$$
|\psi\rangle=\frac{1}{\sqrt{2}}\left(\left|r_{\max }\right\rangle+\left|r_{\min }\right\rangle\right),
$$

where $\left|r_{\max , \min }\right\rangle$ are the eigenvectors of $\rho$ with extreme eigenvalue. This is a coherent superposition of states with extremes eigenvalues fully analogous to an equivalent result for probe optimization for fixed generator [2]. Maybe generators of the form $G \propto|\psi\rangle\langle\psi|$ are rather exotic and void of practical implementations. Nevertheless this example illustrates the fundamental symmetric role of probes and generators.

\section{OPTIMUM NONCLASSICAL STATES}

In this section we show that for three representative generators $G$ (position, number, and number difference) all states providing larger resolution than coherent states are nonclassical, in the sense of lack of positive definite $P(\alpha)$ distribution in Eq. (2.11). 


\section{A. Position operator}

Let us consider transformations generated by the position operator $X$ in Eq. (2.20) that produces the displacement of the conjugate observable $U_{\chi}^{\dagger} Y U_{\chi}=Y+\chi$. Better resolution than the one provided by coherent states $|\alpha\rangle$ (with $a|\alpha\rangle=\alpha|\alpha\rangle$ ) means that

$$
\Lambda^{2}(\rho, X)>\Lambda^{2}(|\alpha\rangle, X)=\left(\Delta_{\alpha} X\right)^{2}=\frac{1}{2} .
$$

By using Eqs. (2.12) and (2.13) we get

$$
\begin{aligned}
\Lambda^{2}(\rho, X) & =\frac{1}{2} \int d^{2} \alpha d^{2} \beta P(\alpha) P(\beta)|\langle\alpha \mid \beta\rangle|^{2} \\
& \times\left\{1+\operatorname{Re}\left[\left(\alpha+\beta^{*}\right)^{2}\right]-\left|\alpha+\beta^{*}\right|^{2}\right\}
\end{aligned}
$$

where Re represents the real part. We have used that $\Lambda^{2}(\rho, X)$ is a real quantity, so that the contribution from the imaginary part of $\left(\alpha+\beta^{*}\right)^{2}$ must vanish.

Condition (3.1) is equivalent to

$$
\int d^{2} \alpha d^{2} \beta P(\alpha) P(\beta) f(\alpha, \beta)<0
$$

where

$f(\alpha, \beta)=1-|\langle\alpha \mid \beta\rangle|^{2}\left\{1+\operatorname{Re}\left[\left(\alpha+\beta^{*}\right)^{2}\right]-\left|\alpha+\beta^{*}\right|^{2}\right\}$,

and we have used that $\int d^{2} \alpha P(\alpha)=1$. Since for all complex numbers $A$ it holds $\operatorname{Re} A \leq|A|$ we get

$$
1+\operatorname{Re}\left[\left(\alpha+\beta^{*}\right)^{2}\right]-\left|\alpha+\beta^{*}\right|^{2} \leq 1
$$

so that $f(\alpha, \beta) \geq 0$ and Eq. (3.3) implies $P(\alpha)<0$ for some $\alpha$. Therefore, the improvement of the intrinsic metrological resolution beyond coherent states implies nonclassical character for the probe.

\section{B. Number operator}

Next we consider transformations generated by the number operator $G=N=a^{\dagger} a$, so that $\chi$ is a phase shift $U_{\chi}^{\dagger} a U_{\chi}=\exp (i \chi) a$. Intrinsic resolution beyond coherent states $|\alpha\rangle$ means

$$
\Lambda^{2}(\rho, N)>\Lambda^{2}(|\alpha\rangle, N)=\left(\Delta_{\alpha} N\right)^{2}=\langle\alpha|N| \alpha\rangle .
$$

In these schemes the accuracy increases when the mean number increases. Therefore, for a proper comparison between the performances provided by different states it is convenient to consider probes with fixed mean number $\operatorname{tr}(N \rho)=\langle\alpha|N| \alpha\rangle$, so that condition (3.6) becomes

$$
\Lambda^{2}(\rho, N)>\operatorname{tr}(N \rho) .
$$

Using the $P(\alpha)$ representation we get

$$
\begin{aligned}
\Lambda^{2} & (\rho, N)=\left(\int d^{2} \alpha d^{2} \beta P(\alpha) P(\beta)|\langle\alpha \mid \beta\rangle|^{2}\right. \\
& \left.\times\left\{\operatorname{Re}\left[\left(\alpha \beta^{*}\right)^{2}\right]-\left|\alpha \beta^{*}\right|^{2}\right\}\right)+\operatorname{tr}\left(N \rho^{2}\right),
\end{aligned}
$$

where again Re represents the real part and we have used that $\Lambda^{2}(\rho, N)$ is a real quantity so that the imaginary part of $\left(\alpha \beta^{*}\right)^{2}$ does not contribute. Since for all $\rho$ it holds $\operatorname{tr}\left(N \rho^{2}\right) \leq \operatorname{tr}(N \rho)$ condition (3.7) implies

$$
\int d^{2} \alpha d^{2} \beta P(\alpha) P(\beta)|\langle\alpha \mid \beta\rangle|^{2}\left\{\operatorname{Re}\left[\left(\alpha \beta^{*}\right)^{2}\right]-\left|\alpha \beta^{*}\right|^{2}\right\}>0 .
$$

Taking into account that $\operatorname{Re} A \leq|A|$ we get

$$
\operatorname{Re}\left[\left(\alpha \beta^{*}\right)^{2}\right]-\left|\alpha \beta^{*}\right|^{2} \leq 0,
$$

so that the fulfillment of condition (3.7) requires nonclassical probes.

\section{Number difference operator}

Finally we consider a two-mode situation with generator $G=J_{z}=N_{1}-N_{2}$ with $N_{j}=a_{j}^{\dagger} a_{j}, j=1,2$, so that in this case $\chi$ is a phase-difference shift $U_{\chi}^{\dagger} a_{1} a_{2}^{\dagger} U_{\chi}=$ $\exp (2 i \chi) a_{1} a_{2}^{\dagger}$. This is perhaps the most common transformation in quantum metrology including all linear interferometric and spectroscopic schemes. Intrinsic resolution beyond the one provided by two-mode coherent states $\left|\alpha_{1}\right\rangle\left|\alpha_{2}\right\rangle$ means that

$$
\Lambda^{2}\left(\rho, J_{z}\right)>\left(\Delta_{\alpha_{1}, \alpha_{2}} J_{z}\right)^{2}=\operatorname{tr}\left[\left(N_{1}+N_{2}\right) \rho\right],
$$

and here again we consider the same total mean number $N_{1}+N_{2}$ in $\rho$ and $\left|\alpha_{1}\right\rangle\left|\alpha_{2}\right\rangle$. In this case

$$
\begin{aligned}
\Lambda^{2}\left(\rho, J_{z}\right) & =\Lambda^{2}\left(\rho, N_{1}\right)+\Lambda^{2}\left(\rho, N_{2}\right) \\
& -2\left[\operatorname{tr}\left(\rho^{2} N_{1} N_{2}\right)-\operatorname{tr}\left(\rho N_{1} \rho N_{2}\right)\right]
\end{aligned}
$$

Denoting by $\boldsymbol{\alpha}=\left(\alpha_{1}, \alpha_{2}\right)$ and $\boldsymbol{\beta}=\left(\beta_{1}, \beta_{2}\right)$ the last term enclosed within square brackets can be rewritten as

$$
\int d^{2} \boldsymbol{\alpha} d^{2} \boldsymbol{\beta} P(\boldsymbol{\alpha}) P(\boldsymbol{\beta})|\langle\boldsymbol{\alpha} \mid \boldsymbol{\beta}\rangle|^{2} f(\boldsymbol{\alpha}, \boldsymbol{\beta}),
$$

where

$$
\begin{aligned}
f(\alpha, \beta)= & \operatorname{Re}\left(\beta_{1}^{*} \alpha_{1} \beta_{2}^{*} \alpha_{2}-\beta_{1} \alpha_{1}^{*} \beta_{2}^{*} \alpha_{2}\right)= \\
& -2 \operatorname{Im}\left(\beta_{1}^{*} \alpha_{1}\right) \operatorname{Im}\left(\beta_{2}^{*} \alpha_{2}\right),
\end{aligned}
$$

so that Eqs. (3.8) and (3.11) imply

$$
\Lambda^{2}\left(\rho, J_{z}\right)=\int d^{2} \boldsymbol{\alpha} d^{2} \boldsymbol{\beta} P(\boldsymbol{\alpha}) P(\boldsymbol{\beta})|\langle\boldsymbol{\alpha} \mid \boldsymbol{\beta}\rangle|^{2} h(\boldsymbol{\alpha}, \boldsymbol{\beta})>0,
$$


where

$$
\begin{aligned}
h(\boldsymbol{\alpha}, \boldsymbol{\beta}) & =\operatorname{Re}\left[\left(\alpha_{1} \beta_{1}^{*}\right)^{2}\right]-\left|\alpha_{1} \beta_{1}^{*}\right|^{2}+\operatorname{Re}\left[\left(\alpha_{2} \beta_{2}^{*}\right)^{2}\right] \\
& -\left|\alpha_{2} \beta_{2}^{*}\right|^{2}+4 \operatorname{Im}\left(\beta_{1}^{*} \alpha_{1}\right) \operatorname{Im}\left(\beta_{2}^{*} \alpha_{2}\right) .
\end{aligned}
$$

By expressing $\alpha_{j} \beta_{j}^{*}$ in terms of their real and imaginary parts, $\alpha_{j} \beta_{j}^{*}=a_{j}+i b_{j}$, we get that $h(\boldsymbol{\alpha}, \boldsymbol{\beta})$ is always negative or zero

$$
h(\boldsymbol{\alpha}, \boldsymbol{\beta})=-2\left(b_{1}-b_{2}\right)^{2} \leq 0 .
$$

Therefore, also for this two-mode generator, resolution beyond coherent states implies nonclassical behavior.

\section{EXAMPLES}

In this section we apply the above formalism to practical probes with Gaussian wave-function (coherent, squeezed, and thermal-chaotic states), and standard generators (phase-space displacements and rotations) looking for optimal intrinsic resolution. Focusing on light beams we consider definite energy resources represented by fixed mean number of photons. Finally we present also the simplest case of a two-dimensional system in order to illustrate some properties of $\Lambda$.

\section{A. Optimum Gaussian states for displacements}

Let us consider signals encoded by $Y$-displacements generated by $X$. Our objective is to obtain maximum intrinsic resolution when using probe Gaussian states with constant energy, i. e., fixed mean number of photons $n$,

$$
n=\operatorname{tr}\left(\rho a^{\dagger} a\right)=\frac{1}{2}\left[\operatorname{tr}\left(\rho X^{2}\right)+\operatorname{tr}\left(\rho Y^{2}\right)-1\right],
$$

which is equivalent to

$n=\operatorname{tr}\left(\rho a^{\dagger} a\right)=\frac{1}{2}\left[(\Delta X)^{2}+(\Delta Y)^{2}+\langle X\rangle^{2}+\langle Y\rangle^{2}-1\right]$,

where $\langle X\rangle,\langle Y\rangle, \Delta X, \Delta Y$, are the corresponding mean values and variances, with $\Delta X \Delta Y \geq 1 / 2$.

Calculations are much simplified if we use the Wigner representation so that the overlap between two states $\rho$, $\rho^{\prime}$ is computed as [11]

$$
\operatorname{tr}\left(\rho \rho^{\prime}\right)=2 \pi \int d x d y W_{\rho}(x, y) W_{\rho^{\prime}}(x, y) .
$$

Under phase-space displacements the Wigner function transforms as a classical distribution

$$
W_{\rho_{\chi}}(x, y+\chi)=W_{\rho}(x, y) .
$$

Furthermore, Wigner functions for Gaussian states are always positive.
Let us consider $\rho$ with Gaussian Wigner function

$$
W_{\rho}(x, y)=\frac{1}{2 \pi \Delta X \Delta Y} \exp \left[-\frac{(x-\langle X\rangle)^{2}}{2(\Delta X)^{2}}-\frac{(y-\langle Y\rangle)^{2}}{2(\Delta Y)^{2}}\right] \text {. }
$$

The Hilbert-Schmidt distance between $\rho_{\chi}$ and $\rho$ for the state (4.5) can be computed exactly

$$
d_{H S}^{2}(\chi)=\frac{1}{\Delta X \Delta Y}\left\{1-\exp \left[-\frac{\chi^{2}}{4(\Delta Y)^{2}}\right]\right\}
$$

Optimum resolution requires minimum $\Delta Y$ for fixed $n$. As reflected by Eq. (4.2) displacement and squeezing compete for the photons since reducing $\Delta Y$ implies increasing $\Delta X$. Since displacement has no effect on the resolution (4.6) optimum results are obtained by employing all photons in squeezing so that $\langle X\rangle=\langle Y\rangle=0$.

For small signals Eq. (4.6) becomes

$$
d_{H S}^{2}(\chi) \simeq \frac{\chi^{2}}{4 \Delta X(\Delta Y)^{3}},
$$

so that

$$
\Lambda^{2}(\rho, X)=\frac{1}{8 \Delta X(\Delta Y)^{3}}
$$

and similarly

$$
\Lambda^{2}(\rho, Y)=\frac{1}{8 \Delta Y(\Delta X)^{3}}
$$

For coherent probes $\Delta X=\Delta Y=1 / \sqrt{2}$ we get $\Lambda^{2}(|\alpha\rangle, X)=1 / 2$. Looking for larger resolutions let us express $\Delta X$ and $\Delta Y$ as functions of the mean energy $n$ and the factor $p=\Delta X \Delta Y \geq 1 / 2$

$$
\begin{aligned}
& (\Delta X)^{2} \simeq n+\sqrt{n^{2}-p^{2}}, \\
& (\Delta Y)^{2} \simeq n-\sqrt{n^{2}-p^{2}},
\end{aligned}
$$

where we have considered $2 n+1 \simeq 2 n$. Let us note that $p$ represents the purity of the probe since $\operatorname{tr}\left(\rho^{2}\right)=$ $1 /(2 p)$. On the other hand, since $\langle X\rangle=\langle Y\rangle=0$ and $(\Delta Y)^{2} \simeq p^{2} /(2 n)$ for $n>>1$, we have that $n$ represents the amount of squeezing for fixed $p$. Using Eq. (44.10) in Eq. (4.8) we get

$$
\Lambda^{2}(\rho, X) \simeq \frac{1}{8 p\left(n-\sqrt{n^{2}-p^{2}}\right)} .
$$

Maximum metrological resolution implies maximum $\Lambda$ which is achieved for minimum $p$, i. e., $p=1 / 2$ (pure minimum uncertainty states), so that for $n>>1$

$$
\Lambda^{2}(\rho, X) \simeq 2 n \text {. }
$$

Therefore the maximum accuracy for displacements of Gaussian probes is obtained for pure nonclassical squeezed vacuum states with

$$
(\Delta Y)^{2} \simeq \frac{1}{8 n}, \quad(\Delta X)^{2} \simeq 2 n
$$




\section{B. Optimum Gaussian states for phase shifts}

In this case we consider signals encoded on Gaussian states with fixed mean energy (mean number $n$ ) by phaseshifts generated by the number operator $N=a^{\dagger} a$. The goal is to obtain maximum intrinsic resolution. Here again we use Gaussian Wigner functions centered at point $\left(x_{0}, 0\right)$ and squeezed along the $y$ direction, so that the overlap under small rotations is minimum,

$$
W_{\rho}(x, y)=\frac{1}{2 \pi \Delta X \Delta Y} \exp \left[-\frac{\left(x-x_{0}\right)^{2}}{2(\Delta X)^{2}}-\frac{y^{2}}{2(\Delta Y)^{2}}\right],
$$

with $\Delta X>\Delta Y$ and

$$
n=\operatorname{tr}\left(\rho a^{\dagger} a\right)=\frac{1}{2}\left[(\Delta X)^{2}+(\Delta Y)^{2}+x_{0}^{2}-1\right] .
$$

Also in this case the Wigner function transforms just by the transformation of its variables, as a classical distribution

$$
W_{\rho_{\chi}}(x, y)=W_{\rho}(x \cos \chi+y \sin \chi, y \cos \chi-x \sin \chi) .
$$

All this leads to the following exact result for the Hilbert-Schmidt distance between $\rho$ and $\rho_{\chi}$

$$
d_{H S}^{2}(\chi)=\frac{1}{\Delta X \Delta Y}\left(1-\frac{\exp A}{\sqrt{B}}\right)
$$

with

$$
A=-\frac{x_{0}^{2} \sin ^{2}(\chi / 2)}{(\Delta X)^{2} \sin ^{2}(\chi / 2)+(\Delta Y)^{2} \cos ^{2}(\chi / 2)},
$$

and

$$
\begin{aligned}
B & =\frac{1}{8(\Delta X)^{2}(\Delta Y)^{2}}\left\{(\Delta X)^{4}+(\Delta Y)^{4}+6(\Delta X)^{2}(\Delta Y)^{2}\right. \\
& \left.-\left[(\Delta X)^{2}-(\Delta Y)^{2}\right]^{2} \cos (2 \chi)\right\} .
\end{aligned}
$$

From Eqs. (2.3) and (4.17) we get

$$
\Lambda^{2}(\rho, N)=\frac{\left[(\Delta X)^{2}-(\Delta Y)^{2}\right]^{2}+2 x_{0}^{2}(\Delta X)^{2}}{16(\Delta X)^{2}(\Delta Y)^{2}} .
$$

From Eq. 4.15), denoting again $p=\Delta X \Delta Y$, we have

$$
\left[(\Delta X)^{2}-(\Delta Y)^{2}\right]^{2}=\left(2 n+1-x_{0}^{2}\right)^{2}-4 p^{2},
$$

and

$$
(\Delta X)^{2}=\frac{1}{2}\left[2 n+1-x_{0}^{2}+\sqrt{\left(2 n+1-x_{0}^{2}\right)^{2}-4 p^{2}}\right] .
$$

Therefore for fixed $n$ and $x_{0}$ the maximum $\Lambda$ is obtained for minimum $p$, i. e., for pure states $p=1 / 2$. Then, the maximum when $x_{0}$ is varied for fixed $n$ is obtained when $x_{0}=0$, leading to

$$
\Lambda^{2}(\rho, N) \simeq n^{2}
$$

which is achieved for the same squeezed vacuum states in Eq. (4.13). The resolution (4.23) is notably larger than the one obtained for coherent states $\Delta X=\Delta Y=1 / \sqrt{2}$ with the same mean number $n=x_{0}^{2} / 2$

$$
\Lambda^{2}(|\alpha\rangle, N) \simeq \frac{n}{2}
$$

\section{Two-dimensional space}

Let us consider an arbitrary Hermitian generator in a two-dimensional space, which can be always expressed in the basis of its eigenvectors as

$$
G=\left(\begin{array}{cc}
g_{1} & 0 \\
0 & g_{2}
\end{array}\right)
$$

An arbitrary probe state reads in the same basis

$$
\rho=\left(\begin{array}{cc}
q & \mu \sqrt{q(1-q)} \\
\mu^{*} \sqrt{q(1-q)} & 1-q
\end{array}\right)
$$

with $1 \geq q \geq 0$ and $|\mu| \leq 1$. In this case we have

$$
\Lambda^{2}(\rho, G)=q(1-q)\left(g_{1}-g_{2}\right)^{2}|\mu|^{2}=|\mu|^{2}\left(\Delta_{\rho} G\right)^{2},
$$

which agrees with the general bound in Eq. (2.14).

We can appreciate that the main difference between $\Lambda(\rho, G)$ and $\Delta_{\rho} G$ is that $\Lambda$ depends on the coherence term $\mu \propto\left\langle g_{1}|\rho| g_{2}\right\rangle$. In particular, for $\mu=0$ we have $\Lambda(\rho, G)=0$ irrespectively of $\Delta_{\rho} G$. This is because in such a case $[\rho, G]=0$ so that $\rho$ is invariant under the transformations generated by $G$. Therefore, although for pure states $\Lambda$ becomes variance, in the general case it is significantly different from a measure of fluctuations.

\section{CONCLUSIONS}

The Hilbert-Schmidt distance is a simple measure of the intrinsic metrological resolution provided by a combination of initial probe state and imprinting transformation. We have shown that for small signals this becomes a probe-transformation measure fully symmetricon the input probe state and on the generator of the transformation. For pure states this coincides with variance, but in the general case it expresses a rather different concept, i. e., metrological resolution. The idea that the probetransformation measure is not always a proper measure of uncertainty is demonstrated by the lack of an uncertainty relation when applying it to complementary generators. We have shown that this is not related with the lack of monotonicity of the Hilbert-Schmidt distance, since the lack of uncertainty relation is reproduced as well by monotonic measures.

Furthermore, we have shown that all states providing resolution larger than coherent states are nonclassical. This is remarkable since this corresponds to states with 
probe-transformation measure larger than for coherent states, while nonclassical behavior is usually ascribed to reduced variances. Nevertheless, for the examples presented in Sec. IV, probe-transformation measure beyond coherent states becomes fully equivalent to reduced variance below the vacuum level.

\section{Acknowledgment}

A.R. acknowledges financial support from the University of Hertfordshire and the EU Integrated Project QAP.
A.L. acknowledges support from the Universidad Complutense Project No. PR1-A/07-15378.
[1] W. M. Itano, J. C. Bergquist, J. J. Bollinger, J. M. Gilligan, D. J. Heinzen, F. L. Moore, M. G. Raizen, and D. J. Wineland, Phys. Rev. A 47, 3554 (1993); M. Hillery and L. Mlodinow, ibid. 48, 1548 (1993); D. J. Wineland, J. J. Bollinger, W. M. Itano, and D. J. Heinzen, ibid. 50, 67 (1994); C. Brif and A. Mann, ibid. 54, 4505 (1996); Z. Y. Ou, ibid. 55, 2598 (1997); V. Giovannetti, S. Lloyd, and L. Maccone, Phys. Rev. Lett. 96, 010401 (2006).

[2] N. D. Mermin, Phys. Rev. Lett. 65, 1838 (1990); J. J. Bollinger, W. M. Itano, D. J. Wineland, and D. J. Heinzen, Phys. Rev. A 54, R4649 (1996); S. F. Huelga, C. Macchiavello, T. Pellizzari, A. K. Ekert, M. B. Plenio, and J. I. Cirac, Phys. Rev. Lett. 79, 3865 (1997); A. Luis, Phys. Rev. A 64, 054102 (2001); 65, 034102 (2002); Ph. Walther, J.-W. Pan, M. Aspelmeyer, R. Ursin, S. Gasparoni, and A. Zeilinger, Nature 429, 158 (2004); M. W. Mitchell, J. S. Lundeen, and A. M. Steinberg, ibid. 429, 161 (2004).

[3] A. Luis, Phys. Rev. A 65, 025802 (2002); Phys. Lett. A 329, 8 (2004); Phys. Rev. A 69, 044101 (2004); ibid. 76, 035801 (2007); J. Opt. B: Quantum Semiclassical Opt. 6, 1 (2004); J. Beltrán and A. Luis, Phys. Rev. A 72, 045801 (2005); S. M. Roy and S. L. Braunstein, e-print arXiv:quant-ph/0607152, S. Boixo, S. T. Flammia, C. M. Caves, and J. M. Geremia, Phys. Rev. Lett. 98, 090401 (2007); S. Boixo, A. Datta, S. T. Flammia, A. Shaji, E. Bagan, and C. M. Caves, Phys. Rev. A 77, 012317 (2008).

[4] S. Luo and Q. Zhang, Phys. Rev. A 69, 032106 (2004).

[5] S. Luo, Phys. Rev. Lett. 91, 180403 (2003).

[6] V. V. Dodonov, O. V. Man'ko, V. I. Man'ko, and A. Wünsche, J. Mod. Opt. 47, 633 (2000); V. V. Dodonov and M. B. Renó, Phys. Lett. A 308, 249 (2003).
[7] J. Lee, M. S. Kim, and Č. Brukner, Phys. Rev. Lett. 91, 087902 (2003); R. Filip, Phys. Rev. A 65, 062320 (2002); M. Hendrych, M. Dušek, R. Filip, and J. Fiurášek, Phys. Lett. A 310, 95 (2003).

[8] V. Vedral, M.B. Plenio, M.A. Rippin, and P.L. Knight, Phys. Rev. Lett. 78, 2275 (1997); V. Vedral and M.B. Plenio, Phys. Rev. A 57, 1619 (1998); C. Witte and M. Trucks, Phys. Lett. A 257, 10 (1998); M. Ozawa, ibid. 268, 158 (2000).

[9] S. A. Ponomarenko and E. Wolf, Opt. Lett. 26, 122 (2001).

[10] S. Chountasis and A. Vourdas, Phys. Rev. A 58, 1794 (1998); S. A. Ponomarenko and E. Wolf, ibid. 63, 062106 (2001); A. Vourdas, ibid. 69, 022108 (2004).

[11] M. Hillery, R. F. O'Connell, M. O. Scully, and E. P. Wigner, Phys. Rep. 106, 121 (1984); M. O. Scully and M. S. Zubairy, Quantum Optics (Cambridge University Press, Cambridge, England, 1997); L. Mandel and E. Wolf, Optical Coherence and Quantum Optics (Cambridge University Press, Cambridge, England, 1995).

[12] Y. Aharanov, D. Z. Albert, and L. Vaidman, Phys. Rev. Lett. 60, 1351 (1988); L. M. Johansen and A. Luis, Phys. Rev. A 70, 052115 (2004).

[13] S. L. Braunstein and C. M. Caves, Phys. Rev. Lett. 72, 3439 (1994).

[14] S. Luo, Proc. Am. Math. Soc. 132, 885 (2003).

[15] S. Luo, Phys. Rev. A 72, 042110 (2005).

[16] This is essentially because Eq. (2.28) is derived in Ref. 5] from a nonpositive definite inner product.

[17] S. Zozor, M. Portesi, and Ch. Vignat, e-print arXiv:math.PR/0709.3011. 